Supplement of Biogeosciences, 17, 667-682, 2020

https://doi.org/10.5194/bg-17-667-2020-supplement

(C) Author(s) 2020. This work is distributed under

the Creative Commons Attribution 4.0 License.

(c) (1)

Supplement of

\title{
Low methane emissions from a boreal wetland constructed on oil sand mine tailings
}

\section{Graham Clark et al.}

Correspondence to: M. Graham Clark (dr.mg.clark@gmail.com)

The copyright of individual parts of the supplement might differ from the CC BY 4.0 License. 


\section{Supplementary information}

Table S1: Published values for methane fluxes from sites with rewetted peatland soils, which also contain a reference to either an undisturbed or an unrestored site.

\section{Seasonal ${ }^{a}$}

\begin{tabular}{|c|c|c|c|c|}
\hline & Sub-site ${ }^{b}$ & $\begin{array}{c}\text { Years since } \\
\text { rewetting }\end{array}$ & $\begin{array}{c}\text { Flux }^{\mathbf{c}} \\
\left(\mathrm{mg} \mathrm{CH}_{4} \mathbf{m}^{-2} \mathbf{h}^{-1}\right)\end{array}$ & $\begin{array}{l}\text { Ratio of } \\
\text { change }^{\text {d }}\end{array}$ \\
\hline \multicolumn{5}{|l|}{ Boreal } \\
\hline \multirow{3}{*}{$\begin{array}{r}\overline{\text { Boreal Plains, Canada }} \\
\text { (Strack et al., 2014) }\end{array}$} & Unrestored - Bare Peat & & $-0.1^{\mathrm{c} 3}$ & $*$ \\
\hline & Rewetted - Dry & 4 & $<0.1^{\mathrm{c} 3}$ & \\
\hline & Rewetted - Wet & 4 & $6^{\mathrm{c} 3}$ & 76.6 \\
\hline \multirow{6}{*}{$\begin{array}{l}\text { Bois-des-Bel } \\
\text { (Waddington and Day, 2007) }\end{array}$} & Unrestored - Cutover (year 1) & & $0.3^{\mathrm{cl}, \mathrm{g}}$ & * \\
\hline & Unrestored - Cutover (year 2) & & $0.1^{\mathrm{cl}, \mathrm{g}}$ & $*$ \\
\hline & Unrestored - Cutover (year 3) & & $0.3^{\mathrm{cl}, \mathrm{g}}$ & $*$ \\
\hline & Rewetted (year 1) & 1 & $<0.1^{\mathrm{cl}, \mathrm{g}}$ & -0.9 \\
\hline & Rewetted (year 2) & 2 & $0.4^{\mathrm{cl}, \mathrm{g}}$ & 2.7 \\
\hline & Rewetted (year 3) & 3 & $1.4^{\mathrm{cl}, \mathrm{g}}$ & 3.6 \\
\hline \multirow{3}{*}{ (Tuittila et al., 2000) } & Unrestored Tussock (year prior to wetting) & & $0.2^{\mathrm{c} 1}$ & $*$ \\
\hline & Restored Tussock & 1 & $0.75^{\mathrm{cl}, \mathrm{f}}$ & 2.8 \\
\hline & Restored Tussock & 2 & $2.5^{\mathrm{cl}, \mathrm{f}}$ & 11.5 \\
\hline \multirow{3}{*}{$\begin{array}{l}\text { Konilamminsuo mire (fen), Sweden } \\
\text { (Komulainen et al., 1998) }\end{array}$} & Unrestored - Low watertable & & $<-0.1$ & * \\
\hline & Rewetted - High watertable & 1 & 1.1 & 132.8 \\
\hline & Rewetted - Average watertable & 1 & 0.2 & 19.8 \\
\hline \multirow{8}{*}{$\begin{array}{l}\text { Multiple Sites, Finland } \\
\text { (Juottonen et al., 2012) }\end{array}$} & Asusuo (Undisturbed) & & $5.8^{\mathrm{cl}}$ & \\
\hline & Kallioneva (Undisturbed) & & $29.5^{\mathrm{c} 1}$ & \\
\hline & Hirsikangas (Undisturbed) & & $9.5^{\mathrm{c} 1}$ & \\
\hline & Mean Undisturbed & & $14.9^{\mathrm{cl}}$ & \\
\hline & Murtsuo (Rewetted) & 11 & $0.3^{\mathrm{c} 1}$ & \\
\hline & Konilamminsuo (Rewetted) & 12 & $0.1^{\mathrm{cl}}$ & \\
\hline & Vanneskorpi (Rewetted) & $10-11$ & $0.5^{\mathrm{c} 1}$ & \\
\hline & Mean Rewetted & & $0.1^{\mathrm{c} 1}$ & -1.0 \\
\hline \multirow{6}{*}{$\begin{array}{l}\text { Sumava National Park, Czech Republic } \\
\text { (Urbanová et al., 2012) }\end{array}$} & Undisturbed Bog - Trichophorum & & 3.8 & \\
\hline & Undisturbed Bog - Shrub & & 3.0 & \\
\hline & Unrestored Bog - Shrub & & 2.2 & $*$ \\
\hline & Unrestored Bog - Molinia & & 0.4 & \\
\hline & Rewetted Bog - Trichophorum & 1 & 2.5 & 0.1 \\
\hline & Rewetted Bog - Shrub Dominated & 1 & 0.4 & -0.8 \\
\hline
\end{tabular}




\begin{tabular}{|c|c|c|c|c|}
\hline & Sub-site ${ }^{b}$ & $\begin{array}{c}\text { Years since } \\
\text { rewetting }\end{array}$ & $\begin{array}{c}\text { Flux }^{\mathbf{c}} \\
\left(\mathbf{m g ~ C H}_{4} \mathbf{m}^{-2} \mathbf{h}^{-1}\right) \\
\end{array}$ & $\begin{array}{l}\text { Ratio of } \\
\text { change }\end{array}$ \\
\hline \multirow{3}{*}{$\begin{array}{l}\text { Viheriäisenneva mire (bog), Sweden } \\
\text { (Komulainen et al., 1998) }\end{array}$} & Unrestored - hollow/lawn & & 0.3 & $*$ \\
\hline & Rewetted - hollow-/awn & 1 & 1.5 & 3.7 \\
\hline & Rewetted - hummock/lawn & 1 & 0.3 & 0.1 \\
\hline \multicolumn{5}{|l|}{ Temperate } \\
\hline \multirow{4}{*}{$\begin{array}{l}\overline{\text { Burns Bog, BC, Canada }} \\
\text { (Christen et al., 2016) }\end{array}$} & Undisturbed & $\mathrm{n} / \mathrm{a}$ & $2.4^{\mathrm{c} 2}$ & \\
\hline & Drained-Sedge/Sphagnum & & $2.8^{\mathrm{c} 2}$ & * \\
\hline & Rewetted-Sedge & 6 & $4.5^{\mathrm{c} 2}$ & 0.6 \\
\hline & Rewetted-Cleared & 2 & $1.6^{\mathrm{c} 2}$ & -0.4 \\
\hline \multirow{3}{*}{ 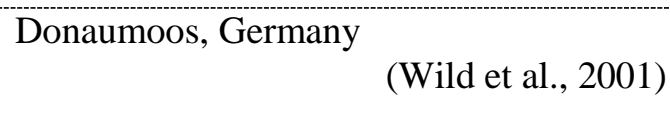 } & Drained - Grassland & & -0.01 & * \\
\hline & Rewetted - Typha & $0.2-1$ & 0.2 & 32.9 \\
\hline & Rewetted - Typha & $0.2-1$ & 1.3 & 131.9 \\
\hline \multirow{2}{*}{$\begin{array}{l}\text { Horstemeer, Netherlands } \\
\text { (Hendriks et al., 2007) }\end{array}$} & Rewetted relatively dry regions & 10 & $2.2 \pm 0.2^{\mathrm{cl}}$ & $*$ \\
\hline & Rewetted-annually saturated & 10 & $18.4 \pm 2.9^{\mathrm{c} 1}$ & 7.3 \\
\hline \multicolumn{5}{|l|}{ Annual $^{a}$} \\
\hline \multicolumn{5}{|l|}{ Boreal } \\
\hline \multirow{6}{*}{$\begin{array}{l}\overline{\text { Ahlen-Falkenberger Moor, Germany }} \\
\text { (Beetz et al., 2013) }\end{array}$} & Natural Wetland (year 1) & & $0.7^{44}$ & \\
\hline & Natural Wetland (year 2) & & $0.4^{44}$ & \\
\hline & Drained - Intensive Management (year 1) & & $<0.1^{\mathrm{c} 4}$ & $*$ \\
\hline & Drained - Intensive Management (year 2) & & $<0.1^{\mathrm{c} 4}$ & $*$ \\
\hline & Rewetted - Extensive Management (year 1) & 3 & $0.2^{\mathrm{c} 4}$ & 9.5 \\
\hline & Rewetted - Extensive Management (year 2) & 4 & $<0.1^{\mathrm{c} 4}$ & 2.6 \\
\hline \multicolumn{5}{|l|}{ Temperate } \\
\hline \multirow{4}{*}{$\overline{\text { Ballacorick, Ireland }}$ (Wilson et al., 2013) } & Rewetted - Bare Peat (relatively Dry) & Mean of 7-9 & $<0.1$ & $*$ \\
\hline & Rewetted - Juncus/Spagnum & Mean of 7-9 & 1.2 & 72.1 \\
\hline & Rewetted - Sphagnum & Mean of 7-9 & 1.5 & 87.4 \\
\hline & Rewetted-Euiophorum & Mean of 7-9 & 0.8 & 46.3 \\
\hline \multirow{4}{*}{$\begin{array}{l}\text { Glenvar, Ireland } \\
\text { (Renou-Wilson et al., 2016) }\end{array}$} & Unrestored - Grazing allowed & & $0.2^{\mathrm{c} 4}$ & $*$ \\
\hline & Unrestored - No Grazing & & $0.3^{\mathrm{c}}$ & $*$ \\
\hline & Rewetted - Grazing allowed & $15-16$ & $1.9^{\mathrm{c}}$ & 6.8 \\
\hline & Rewetted - No Grazing & $17-18$ & $0.9^{\mathrm{c}}$ & 2.0 \\
\hline \multirow{4}{*}{$\begin{array}{l}\text { Himmelmoor, Germany } \\
\text { (Vanselow-Algan et al., 2015) }\end{array}$} & Unrestored - Extraction site & & $<0.1^{\mathrm{c} 1}$ & $*$ \\
\hline & Restored Heath dominated & $3-30$ & $7.2^{\mathrm{cl}}$ & 201.0 \\
\hline & Restored Sphagnum dominated & $3-30$ & $11.4^{\mathrm{c} 1}$ & 315.0 \\
\hline & Restored Purple moor grass dominated & $3-30$ & $17.0^{\mathrm{c} 1}$ & 470.1 \\
\hline
\end{tabular}




\begin{tabular}{|c|c|c|c|c|}
\hline & Sub-site $^{\mathrm{b}}$ & $\begin{array}{l}\text { Years since } \\
\text { rewetting }\end{array}$ & Flux $^{\mathrm{c}}$ & $\begin{array}{l}\text { Ratio of } \\
\text { change }^{d}\end{array}$ \\
\hline \multirow{3}{*}{$\begin{array}{l}\text { Horstermeer, Netherlands } \\
\text { (Hendriks et al., 2007) }\end{array}$} & Rewetted - Relatively Dry & 10 & 2.3 & $*$ \\
\hline & Rewetted - Wet & 10 & 18.4 & 7.11 \\
\hline & Ditch & 10 & 9.1 & 3.0 \\
\hline \multirow{4}{*}{$\begin{array}{l}\text { Schleswing-Holstein, Germany } \\
\text { (Poyda et al., 2016) }\end{array}$} & Drained - Arable land & & $<0.1$ & $*$ \\
\hline & Drained - Moist, agricultural production & & $<0.1$ & -0.5 \\
\hline & Drained - Wet, agricultural production & & 0.2 & 6.6 \\
\hline & Rewetted & Mean of 20-23 & 0.8 & 30.1 \\
\hline \multicolumn{5}{|l|}{ Peak flux ${ }^{a}$} \\
\hline \multicolumn{5}{|l|}{ Boreal } \\
\hline 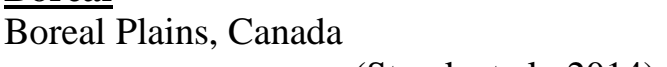 & Drained - Extraction & & -0.05 & * \\
\hline (Strack et al., 2014) & Rewetted & 4 & 16.4 & 350.9 \\
\hline \multicolumn{5}{|l|}{ Temperate } \\
\hline \multirow[t]{4}{*}{ Turraun, Ireland } & Unrestored & 0 & $0.1^{\mathrm{e}}$ & \\
\hline & Restored-Typha & $10-14$ & 16 & 159 \\
\hline & Restored-Phalaris & $10-14$ & $6^{\mathrm{e}}$ & 59 \\
\hline & Restored-Eriophoum/Carex & $10-14$ & 1.8 & 17 \\
\hline
\end{tabular}

5 a - Literature reported seasonal, annual fluxes, or maximum. Scaled to hourly fluxes over the study period for consistency of units used in this study.

b - Sub-sites are labelled with either dominate vegetation, or treatment as worded within each paper.

c - c1 designates mean, 22 designates median, c3 designates midpoint of the given range, c4 designates annual total divided by 8760 hours.

d - \% change is the difference between the rewetted site flux and the dry site flux divided by the absolute dry site flux. i.e. the effect of wetting on methane emissions. *indicates which subsite was used for the dry site flux.

10 e - Values interpreted from figure 2 in Wilson et al. 2009.

f - value interpreted from figure 2 in Tuttila et al 2000.

g - spatially weighted mean flux from table 2 values in Waddington and Day 2007. 


\section{References}

Beetz, S., Liebersbach, H., Glatzel, S., Jurasinski, G., Buczko, U., Höper, H., 2013. Effects of land use intensity on the full greenhouse gas balance in an Atlantic peat bog. Biogeosciences 10, 1067-1082.

Christen, A., Jassal, R.S., Black, T.A., Grant, N.J., Hawthorne, I., Johnson, M.S., Lee, S.-C., Merkens, M., 2016. Summertime greenhouse gas fluxes from an urban bog undergoing restoration through rewetting. Mires Peat 17, 1-24.

Hendriks, D.M.D., van Huissteden, J., Dolman, a. J., van der Molen, M.K., 2007. The full greenhouse gas balance of an abandoned peat meadow. Biogeosciences 4, 277-316.

20 Juottonen, H., Hynninen, A., Nieminen, M., Tuomivirta, T.T., Tuittila, E., Nousiainen, H., Kell, D.K., 2012. Methane-Cycling Microbial Communities and Methane Emission in Natural and Restored Peatlands. Appl. Environ. Microbiol. 78, 63866389.

Komulainen, V.M., Nykanen, H., Martikainen, P.J., Laine, J., 1998. Short-term effect of restoration on vegetation change and methane emissions from peatlands drained for forestry in southern Finland. Can. J. For. Res. 28, 402-411.

25 Poyda, A., Reinsch, T., Kluß, C., Loges, R., Taube, F., 2016. Greenhouse gas emissions from fen soils used for forage production in northern Germany. Biogeosciences 13, 5221-5244.

Renou-Wilson, F., Müller, C., Moser, G., Wilson, D., 2016. To graze or not to graze? Four years greenhouse gas balances and vegetation composition from a drained and a rewetted organic soil under grassland. Agric. Ecosyst. Environ. 222, 156170.

30 Strack, M., Keith, A.M., Xu, B., 2014. Growing season carbon dioxide and methane exchange at a restored peatland on the Western Boreal Plain. Ecol. Eng. 64, 231-239.

Tuittila, E., Komulainen, V., Vasander, H., Nykanen, H., Martikainen, P.J., Laine, J., 2000. Methane dynamics of a restored cut-away peatland. Glob. Chang. Biol. 6, 569-581.

Urbanová, Z., Picek, T., Hájek, T., Bufková, I., 2012. Vegetation and carbon gas dynamics under a changed hydrological regime in central European peatlands. Plant Ecology \& Diversity, 5(1), 89-103.

Vanselow-Algan, M., Schmidt, S.R., Greven, M., Fiencke, C., Kutzbach, L., Pfeiffer, E.M., 2015. High methane emissions dominated annual greenhouse gas balances 30 years after bog rewetting. Biogeosciences 12, 4361-4371.

Waddington, J.M., Day, S.M., 2007. Methane emissions from a peatland following restoration. J. Geophys. Res. 112, 1-11.

Wild, U., Kamp, T., Lenz, A., Heinz, S., 2001. Cultivation of Typha spp . in constructed wetlands for peatland restoration. Ecol. Eng. 17, 49-54.

Wilson, D., Alm, J., Laine, J., Byrne, K.A., Farrell, E.P., Tuittila, E.S., 2009. Rewetting of cutaway peatlands: Are we recreating hot spots of methane emissions? Restor. Ecol. 17, 796-806.

Wilson, D., Farrell, C., Mueller, C., Hepp, S., 2013. Rewetted industrial cutaway peatlands in western Ireland : a prime location for climate change mitigation? Mines Peat 11, 1-22. 